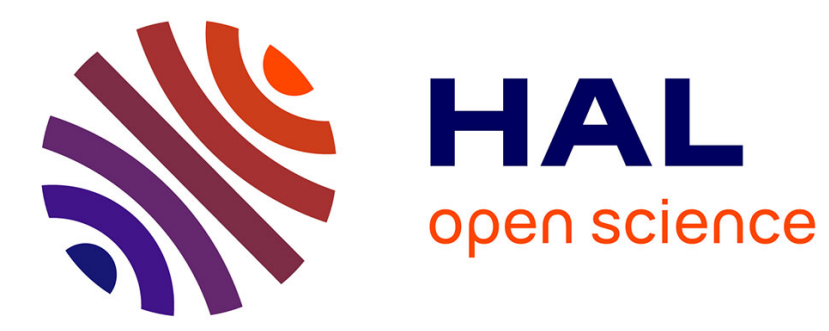

\title{
Nanoscale surface topography reshapes neuronal growth in culture
}

Ghislain Bugnicourt, Jacques S. Brocard, Alice Nicolas, Catherine Villard

\section{To cite this version:}

Ghislain Bugnicourt, Jacques S. Brocard, Alice Nicolas, Catherine Villard. Nanoscale surface topography reshapes neuronal growth in culture. Langmuir, 2014, 30 (15), pp.4441. 10.1021/la5001683 . hal-00987842

\section{HAL Id: hal-00987842 \\ https://hal.science/hal-00987842}

Submitted on 25 Jun 2014

HAL is a multi-disciplinary open access archive for the deposit and dissemination of scientific research documents, whether they are published or not. The documents may come from teaching and research institutions in France or abroad, or from public or private research centers.
L'archive ouverte pluridisciplinaire HAL, est destinée au dépôt et à la diffusion de documents scientifiques de niveau recherche, publiés ou non, émanant des établissements d'enseignement et de recherche français ou étrangers, des laboratoires publics ou privés. 


\title{
Nanoscale surface topography reshapes neuronal growth in culture
}

\author{
Ghislain Bugnicourt ${ }^{1,2}$, Jacques Brocard ${ }^{2}$, Alice Nicolas ${ }^{3}$, Catherine Villard ${ }^{1}$ \\ E-mail : catherine.villard@neel.cnrs.fr (corresponding author) \\ ${ }^{1}$ Univ. Grenoble Alpes, Inst NEEL and CRETA, F-38042 Grenoble, France. \\ CNRS, Inst NEEL and CRETA, F-38042 Grenoble, France \\ ${ }^{2}$ Institut National de la Santé et de la Recherche Médicale, U836-GIN, 38700 Grenoble Cedex \\ 9 (France) \\ ${ }^{3}$ CNRS-LTM-UJF, CEA-LETI 17 Av. des Martyrs 38054 Grenoble FRANCE
}

\begin{abstract}
Neurons are sensitive to topographical cues provided either by in vivo or in vitro environments on the micrometric scale. We have explored the role of randomly distributed silicon nano-pillars on primary hippocampal neurite elongation and axonal differentiation. We observed that neurons adhere on the upper part of nano-pillars with a typical distance between adhesion points of about 500nm. These neurons produce less neurites, elongate faster, and differentiate an axon earlier than those grown on flat silicon surfaces. Moreover, when confronted to a differential surface topography, neurons specify an axon preferentially onto nano-pillars. As a whole, these results highlight the influence of the physical environment in many aspects of neuronal growth.
\end{abstract}




\section{Introduction}

In vitro neurons are usually plated on glass coverslips or petri dishes. However, these substrates provide to developing neurons a flat and uniform environment that contrasts with the complex tri-dimensional topography of the embryonic brain ${ }^{1}$. Moreover, cellular adhesion involves adhesive complexes mediated by transmembrane heterodimers, named integrins, that are established on the micrometric ${ }^{2}$ and ever sub-micrometric scale ${ }^{3}$. Understanding the mechanisms of cell adhesion might therefore benefit from the use of micro structured surfaces.

In line with this reasoning, the specific interaction between neurons and different micro-pillar geometries has been explored. A common feature observed on these pillared surfaces is a neurite channeling effect between pillars when the inter-pillar spacing is larger but close to the neurite width $(\approx 1-2 \mu \mathrm{m})^{4-6}$.Another remarkable effect is the accelerated neurite elongation provided by micro-pillars ${ }^{4,6}$. Interestingly, axons have been reported to specify preferentially within micro-pillars areas compared to control flat surfaces ${ }^{6}$, a feature also observed when micro-pillars are replaced by submicroscale holes ${ }^{7}$. Another generic behavior is the transition between a neurite channeling effect between pillars to a neurite growth on pillars. This transition seems to occur for inter-pillar distances lower than the neurite width ${ }^{5-6}$, possibly due to geometrical arguments.

A stringent restriction of elementary adhesive areas up to the nanometric scale was also explored. Spatz and Geiger used regularly spaced adhesive gold nanoparticles to demonstrate that cell spreading is an active process controlled by density-dependent integrin signals ${ }^{8}$. The role of tri-dimentional topographies on the nanometer scale has been also studied using GaP vertical nanowires (GaP-NWs) with typical diameters in the range 50-80nm. Depending on the inter-NW distance, channeling effect ${ }^{9}$, neurite growth on top of GaP-NWs bi-dimentional networks $^{10}$, or neurite guidance above GaP-NWs rows were reported ${ }^{11}$. Growth on top of GaP-NWs was observed when the distance between GaP-NWs was about half a micron.

Beside these fundamental aspects of neurobiology that ultimately might concern the issue of neuro-regeneration, acquiring knowledge about the interaction between neurons and nanostructured surfaces meets the requirements of recent intra-cellular recording techniques based on the insertion of vertical nanostructures through cell membranes ${ }^{12-13}$.

Following the studies on the interaction between GaP nanowires and either retinal ${ }^{10}$ or cervical and dorsal root ganglia neurons ${ }^{9,11}$, the present work explore in details the role of randomly distributed silicon nano-pillars on primary hippocampal neurite elongation rate and 
axonal polarization. In the course of our study, we were led to discriminate between the role of topography and effective rigidity, and to evaluate the directional choices performed by developing neurons according to the spatial distribution of nano-pillars. How neurons behave at the frontier between nano-structured and flat surfaces was also explored. Our results reinforce the growing body of evidences of the role of topographical cues at the submicron scale, and show that spatially distributed nanometric adhesive areas over submicron distances influences many aspects of neuronal growth during the first stages of development. 


\section{Materials and methods}

\section{1 - Structuration of silicon surfaces.}

Nanopillared surfaces were prepared by reactive ion etching performed on silicon substrates cut from $51 \mathrm{~mm}$ in-diameter wafers (four substrates per wafer). This $50 \mathrm{~W}$ etching process involves $\mathrm{SF}_{6}$ gas (64\% in volume) and $\mathrm{O}_{2}(36 \%)$. The oxygen-based plasma forms a silicon oxide layer on the exposed Si surface while the SF6-based plasma etches both the silicon and the silicon oxide $\left(\mathrm{SiO}_{2}\right)$, the latter with a lower speed. The combination of these two gases gives rise to a competition between etching and passivation. This complex phenomenon after about 15 minutes produces a carpet of silicon nano-pillars capped by a $\mathrm{SiO}_{2}$ layer and spaced by sub-micrometric distances.

Etching was not uniformly performed on the whole sample surface but restricted to rectangular, millimeter-sized, areas (see Fig. S1 in the Supporting Material). This large scale modulation of the substrate topography was achieved by classical UV photolithography steps including Shipley S1818 photoresist spinning (4000 rpm, $1.8 \mu \mathrm{m}$ thickness, $115^{\circ} \mathrm{C}$ annealing step for $1 \mathrm{~min}$ ), insulation through a mask and development (Microposit concentrate 1:1, Shipley). After the etching process, the remaining layer of photoresist protecting the silicon surface was removed in pure acetone. Then, a pure oxygen plasma $(2 \mathrm{~min}, 50 \mathrm{~W})$ was used to obtain hydrophilic surface properties suitable for the next step of cellular functionalization of both pillared and flat silicon surfaces.

\section{2 - Neuronal culture and labeling}

Mouse hippocampal neurons (E18.5) were prepared and seeded over Poly-L-Lysine-covered surfaces as previously described ${ }^{13}$. Poly-L-lysine at a concentration of $1 \mathrm{mg} / \mathrm{ml}$ incubated overnight at room temperature was used for cell adhesion. In some experiments, laminin coating was also used at a concentration of $10 \mu \mathrm{g} / \mathrm{ml}$ incubated 6 hours at $37{ }^{\circ} \mathrm{C}$ after the deposition of Poly-L-lysine.

Two types of fixation protocols were used depending on the imaging technique. For optical observations, we used paraformaldehyde (PFA) according to the following protocol: (i) the plates were incubated at $37^{\circ} \mathrm{C}$ for about half an hour with a solution of PFA / Sucrose (paraformaldehyde 4\%, $120 \mathrm{mM}$ sucrose, PBS), then (ii) after washing in PBS, cells were permeabilized for a few minutes in PBS / Triton X-100 0.1\%, and (iii) Triton was then eliminated by rinsing with PBS. For scanning electron microscopy, the PFA protocol was used with addition of glutaraldehyde $0.5 \%$ and without the step of membrane 
permeabilization (i.e. without Triton). The sample was then dehydrated by successive few minutes dipping in 50\% (diluted with water) then $100 \%$ acetone followed by immersion in $50 \%$ (diluted with acetone) then $100 \%$ hexamethyldisilazane (HMDS) ${ }^{14}$. Samples were finally allowed to dry slowly under a hood.

Primary antibodies were Tau (clone tau-1, Millipore), rat mAb against tubulin (cloneYL1/2) and mouse anti-vinculin (Sigma). Secondary antibodies were Alexa488 or Cy3 coupled (Molecular Probes, USA). Neurons were observed with two different microscopes: a Zeiss Ultra Scanning Electron Microscope and a BX51 optical microscope (Olympus, Inc.) using either 10x, 20x or 40x dry objectives combined with a F-View II camera.

\section{3- Image analysis}

Automatic measurements were performed using the free software Image ${ }^{15}$ with custom-made plugins specifically developed for this study.

Silicon topography: Scanning electron microscope (SEM) images from top view (magnification: 24kX, polaroid reference) were first processed in ImageJ ("smooth", "threshold", "remove outliers") and then loaded into Gwyddion ${ }^{16}$ to extract pillars coordinates and radius. The coordinates were further analyzed in ImageJ by calculating all the distances for each pillar and sorting them in ascending order to get the $\mathrm{n}^{\text {th }}$ neighbor distances. Note that these $n^{\text {th }}$ neighbor distances were kept only for the pillars situated in the center of the image (in a square whose surface is a fourth of the total square image) to avoid side-effects.

For straight line distances, five horizontal lines (one pixel thick) were drawn arbitrarily on the image. Around four pillars were found along each line, but only the first inter-pillar distance was taken into account. The root square of the mean distances was calculated to obtain a normal distribution, whose mean was used as the representative line distance. A similar method using the mean value of the distribution of the root square distances was employed to compute the adhesion point distance.

Other lengths, surfaces and angles were measured manually using the segmented line tool of ImageJ.

Cellular lengths: the automatic measurements developed for the needs of this study consist in (i) thresholding the image of microtubules in fluorescence, then (ii) skeletonizing the signal by the method ${ }^{17}$ to transform both neurites and somas into white lines of 1-pixel thickness on a black background, and finally (iii) setting the lines width to three pixels with the ImageJ function "dilate" for binary images. The total length of cells in a given image is then obtained 
by dividing the total white area by three. Of note, this method was proven to make fewer errors in the evaluation of neurite length than 1-pixel skeletonization compared to manual measurements. The number of cells in the image is determined by manual counting. Only optical micrographs with reasonable cell densities, between 7 and 16 neurons per field of $892 \times 673 \mu \mathrm{m}^{2}$ (about $0.6 \mathrm{~mm}^{2}$ ) were used.

Finally, the ratio of the total cell length on the number of cells gives the average neurite length per cell. Note that although many cells have neurites coming out from the image, there is statistically as many neurites entering the same optical field.

\section{4-Statistics}

All percentage comparisons were performed using $\chi^{2}$ tests. Quantitative measurements were analyzed via a Kolmogorov-Smirnov test to assess normality, then compared using a standard unpaired t-test. All the calculations were performed on Excel (Microsoft). 


\section{Results}

\section{1 - Topographical characteristics of silicon surfaces.}

The etching process changed the initial flat silicon surface into a nanopillared surface (Figure la, top) defined by an isotropic (see Fig. S2a-c) pillar density of about 3 per $\mu \mathrm{m}^{2}$ (i.e. $300 \times 10^{6}$ nano-pillars per $\mathrm{cm}^{2}$ ) and statistically distributed characteristic inter-pillar distances (Figure 1b). We estimated these distances according to two different methods. The « first neighbor distance » is a usual physical parameter that gives the average distance to the closest pillar (see Fig. S2d in the Supporting Material for the example of a serie of $n^{\text {th }}$ neighbor distances). The " line distance » assesses the average distance between a pillar and the first other pillar met along a straight line evocating the directional persistency of neurites, and of axons in particular $^{18}$. The first neighbor distance $(210 \pm 60 \mathrm{~nm})$ is the lowest one (with $890 \pm 150 \mathrm{~nm}$ for the " line distance », Figure 1b). Typically, the nano-pillars adopt a tapered shape characterized by a typical height of $700 \mathrm{~nm}$ and a main radius of $35 \pm 17 \mathrm{~nm}$. The formation of this surface topography is accompanied by a digging of the bulk silicon, leading to the presence of a step at the border between flat and nano-structured surfaces (Figure 1a, bottom).

a
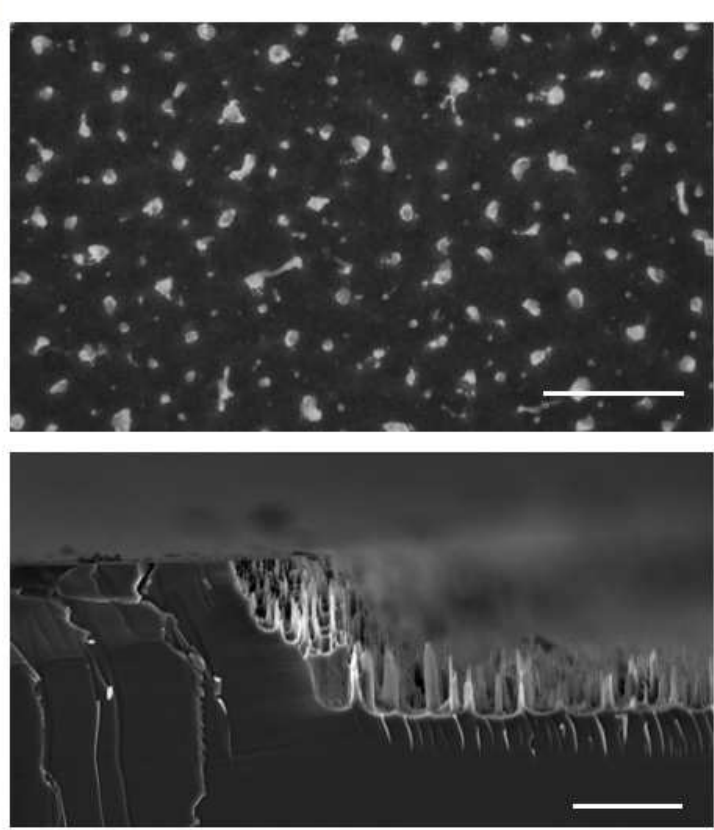

b
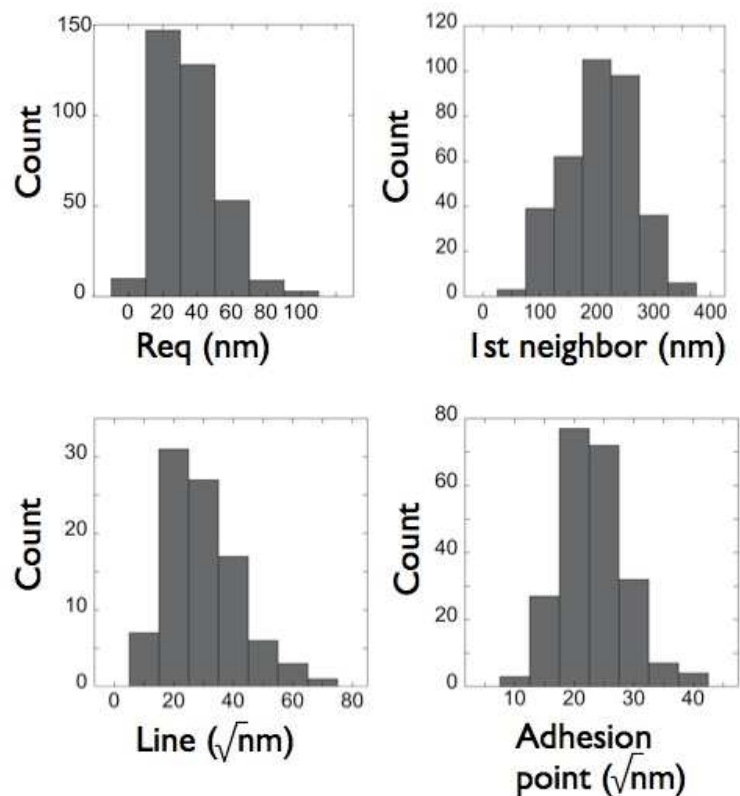

Figure 1 - Morphology and characteristic lengths of silicon nanopillared surfaces.

a) SEM micrographs (top view and section). Scale bars: $500 \mathrm{~nm}$.

b) Histograms of the equivalent radius of the nano-pillars (Req) and of the different characteristic distances between nano-pillars. The mean values of the distributions are indicated between brackets. Req $(35 \pm 17 \mathrm{~nm}, \mathrm{n}=350)$, first neighbor $(210 \pm 60 \mathrm{~nm}, \mathrm{n}=349)$, 
square root of the distance between nano-pillars in a given arbitrary direction (i.e in our case along an horizontal line, 29.67 $\pm 12.24 \sqrt{\mathrm{nm}}, \mathrm{n}=92$ ) and under a neurite (adhesion points, $22.96 \pm 5.21 \sqrt{\mathrm{nm}}, \mathrm{n}=222$ ) (for all values: mean \pm standard deviation of the distribution, with $\mathrm{n}$ denoting the number of measured distances).

Three silicon substrates (see methods) were analyzed. The number of images used for these analysis were 20, 20 and 24 for the first neighbour, line and adhesion point distances, respectively.

\section{2 - Neurons form adhesions on the upper part of nano-pillars.}

We observed, as expected from the submicrometric values of the inter-pillar distances, that neurons did not develop at the basis of the nano-pillars. The cells rather display adhesive contacts close to the free extremities of the nano-pillars by clinging either to their tops or to their top edges. This leads to the discretization of their adhesion both at the soma and at the neurite levels (Figure 2a). The mean distance between neurite adhesive contacts is $527 \pm 27$ $\mathrm{nm}$, an intermediate value between the « first neighbor » and « line » distances (Figure 1b). This indicates that growing neurites cannot bend enough to select the closest peak neither grow straight, but instead make directional choices. 

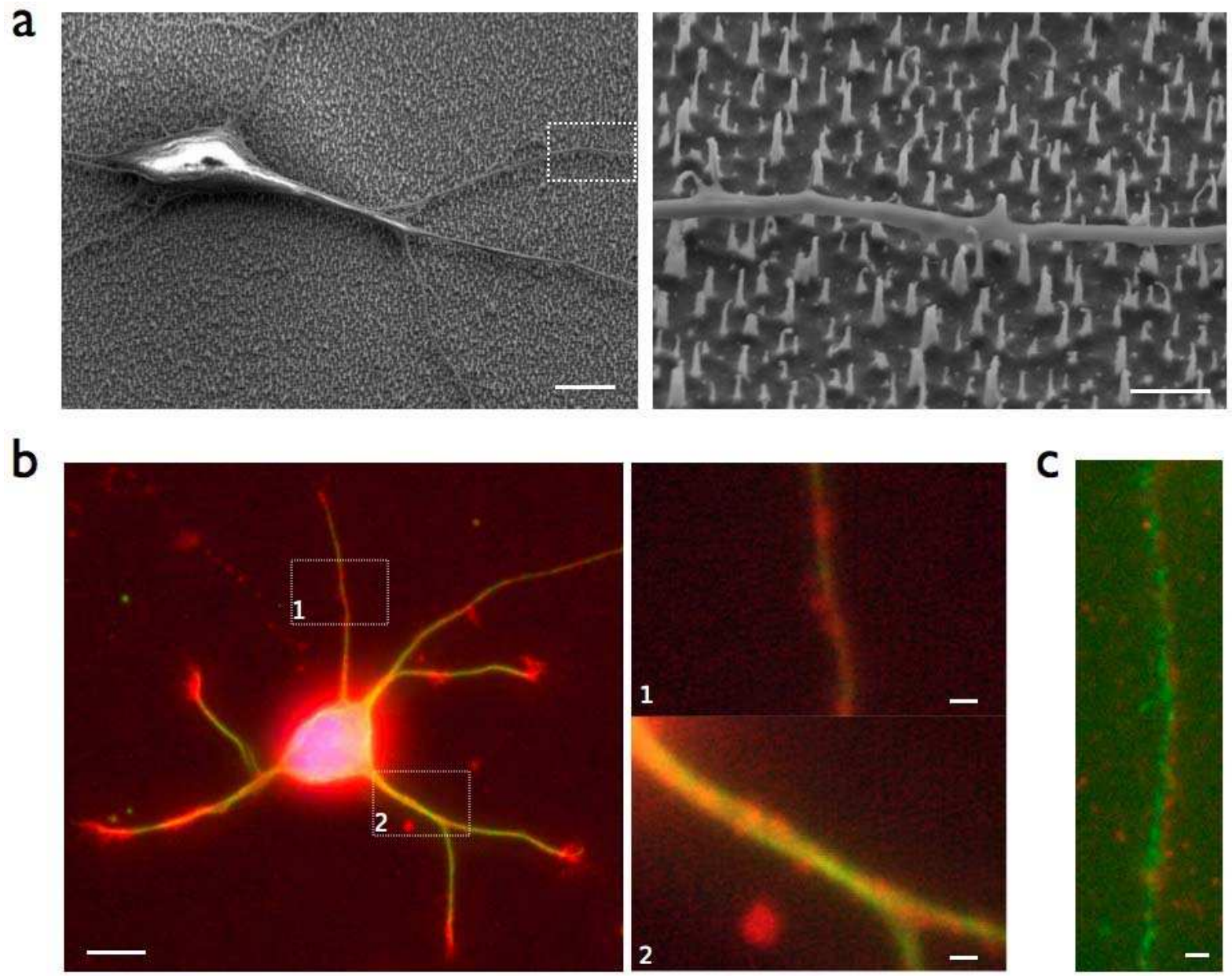

Figure 2 - Neurons adhere on the upper part of nano-pillars

a) SEM micrographs of a neuron after fixation and dehydration. Right: high magnification view of the area boxed on the left image. Scale bars: $5 \mu \mathrm{m}$ (left) and $1 \mu \mathrm{m}$ (right).

b) Actin (Phalloïdin, red), microtubules (YL1/2, green) and nucleus (Hoechst, blue) immunolabelling. The details of the actin structure are displayed on the high magnification views of the areas boxed on the left image. Scale bars : $10 \mu \mathrm{m}$ (left) and $1 \mu \mathrm{m}$ (right).

c) Actin (Phalloïdin, red) and vinculin (anti-vinculin, green) immunolabelling along a neurite. The two colored images are slightly shifted to reveal each staining separatly. Scale bar: $2 \mu \mathrm{m}$.

We decided to build a probabilistic model to quantify this directional choice. This model should answer the question « what would be the mean distance $L_{\alpha}$ between two adhesion points if the probability to find the next nano-pillar is restricted to an angle $\alpha » L_{\alpha}$ can be written as a series of terms expressing each the probability, weighted by the distance to the $n^{\text {th }}$ neighbor $\left(D_{n}\right)$, to find a pillar in an arc $\alpha$ knowing that no pillar has been found for the previous (n-1) neighbours (see Fig. 3a for a representation of these parameters). Knowing that 
the probability to find a peak in an arc of angle $\alpha$ is the ratio $\frac{\alpha}{2 \pi}$ the first term of the mathematical serie is $L_{1}=\frac{\alpha}{2 \pi} \times D_{1}$. The second term can be written as $L_{2}=\left(1-\frac{\alpha}{2 \pi}\right) \frac{\alpha}{2 \pi} \times D_{2}$, i.e. as the probability $\left(1-\frac{\alpha}{2 \pi}\right)$ of not finding the first neighbor in an arc of angle $\alpha$ multiplied by the probability $\frac{\alpha}{2 \pi}$ to find the second-neighbor in an arc of angle $\alpha$. Similarly, the third term is $L_{3}=\left(1-\frac{\alpha}{2 \pi}\right)^{2} \times \frac{\alpha}{2 \pi} \times D_{3}$, leading to the expression for the whole series:

$$
L_{\alpha}=\sum_{n=1}^{\infty} L_{n}=\sum_{n=1}^{\infty}\left(1-\frac{\alpha}{2 \pi}\right)^{(n-1)} \times \frac{\alpha}{2 \pi} \times D_{n}
$$

Then, the series of the $\mathrm{n}^{\text {th }}$ neighbor distances $D_{n}$ and the mean distance between adhesion points (expressed as $L_{\alpha}$ in equation 1) were measured in the same areas, and the value of $\alpha$ assessed numerically by equalizing the two terms of equation 1 . From the analysis of a large number of images ( $\mathrm{n}=44$, leading to $\mathrm{n}$ values of $L_{\alpha}$, themselves calculated from an average of 20 distances between adhesion points) we obtained the histogram shown in Figure $\mathbf{3 b}$.
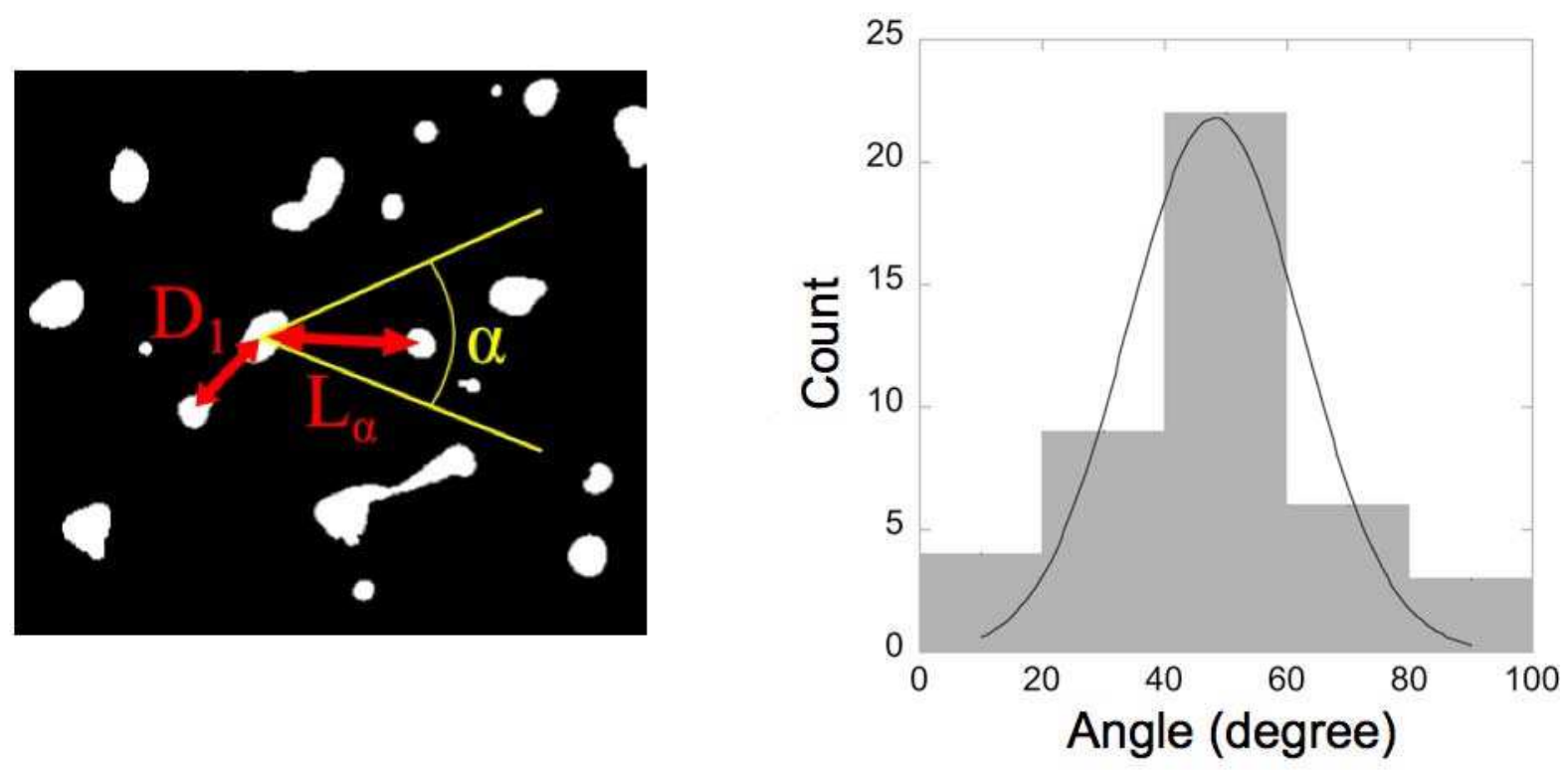

\section{Figure 3 - Neurite directional choices.}

a) Zoom on a binarized top-view of the rough surface. The first neighbor distance $D_{1}$ is sketched together with $L_{\alpha}$ that represents the distance to the first neighbor in an angle $\alpha$ (in an arbitrary direction since the peak distribution is isotropic). This angle can be calculated based on the $D_{n}$ values and the experimental distances $L_{\alpha}$ between adhesive points (see equation 1). 
b) Histogram of the distribution of the angle $\alpha$ expressing the angular tolerance to bending of neurites developing on top of nano-pillars. Each count corresponds to the analysis of one SEM micrograph providing in average 20 values of distances between adhesion points $(\mathrm{n}=44)$. The curve is a gaussian fit of the data, leading to $\alpha=48^{\circ} \pm 20^{\circ}$ (standard deviation).

The most probable value of $\alpha$ is $48^{\circ} \pm 20^{\circ}$ (standard deviation). At the scale of adhesions, the neurite thus tolerates an angle of about $25^{\circ}$ to bend on either side of the mean trajectory. This means that the neurite would stretch and eventually unhook from nano-pillars too distant from the average trajectory if the path followed by the growth cone would lead to a greater neurite curvature. Interestingly, the value of $25^{\circ}$ is very close to the range of orientational changes in the growth direction of chick embryos and Xenopus axons in a time scale of $10 \mathrm{~min}$, as reported by $\mathrm{Katz}^{18}$.

Growth cones themselves adhere on top of the nano-pillars. To see if this discontinuous adhesive surface had an effect on their shape, we labeled actin filaments (F-actin) with phalloidin to reveal growth cones at the neurite tips. Interestingly, no differences were observed in the general shape between nano-pillars and flat surfaces: growth cones presented very similar surfaces, fluorescence intensities and the percentage of active growth cones (i.e. the percentage of neurite tip ending with a large actin structure evocative of a growth cone) among all neurite extremities was unchanged (Fig. S3).

Of note, actin and vinculin labelings show a discrete distribution of these proteins characterized by the presence of spots separated by sub-micrometer distances (Figure $\mathbf{2 b}$ and 2c). This may indicate the existence of adhesion complexes around peaks as evidenced by the presence of vinculin, a signaling protein which couples integrin receptors to actin filament, and $\operatorname{actin}^{11,19}$.

In brief, we achieved a discontinuous adhesive environment for neuronal growth, characterized by point-like adhesions of the typical size of elementary integrin structures separated by distances on the order of magnitude of the neurite diameter. Moreover, the macroscopic alternation of nano-pillared and flat areas created frontiers along which individual neuron can explore simultaneously two different physical environments. We therefore studied how neurons developed on silicon nano-pillared compared to flat surfaces and how they located their axons when confronted to a differential surface topography.

\section{3- Influence of nano-pillars on neuronal development}




\section{1 - Neuronal development at 3DIV (Days In Vitro)}

We focused on three morphological parameters that are the total neurite length, the number of neurites emerging from the soma, and the mean neuritic length that is obtained by dividing the total length by the neurite number. The graphs of Figure 4, in which each point results from the analysis of one field of about $0.6 \mathrm{~mm}^{2}$ (Figure 4a), present a comparison of neuronal growth on nano-pillared and flat areas at 3DIV. It appears that neurons are more developed on nano-pillars (total neuritic length of $636 \pm 165 \mu \mathrm{m}$ compared to $506 \pm 142 \mu \mathrm{m}, \mathrm{p}<0.001$, ***), produce less neurites $(3.6 \pm 0.5$ compared to $4.6 \pm 0.7, \mathrm{p}<0.001, * * *)$, and therefore present a longer mean neurite length $(180 \pm 47 \mu \mathrm{m}$ compared to $112 \pm 32 \mu \mathrm{m}, \mathrm{p}<0.001$, ***). Note that our control condition (flat silicon surfaces covered by a native silicon oxide) gave results very similar to other studies on glass substrates ${ }^{20}$, as expected from their similar surface chemistry. Beside, the reduced neurite number on nanopillared surfaces raises the question of what happens at earlier stages of cell spreading and development. We therefore conducted a similar study at $2 \mathrm{DIV}$, then only 10 hours after plating.

a
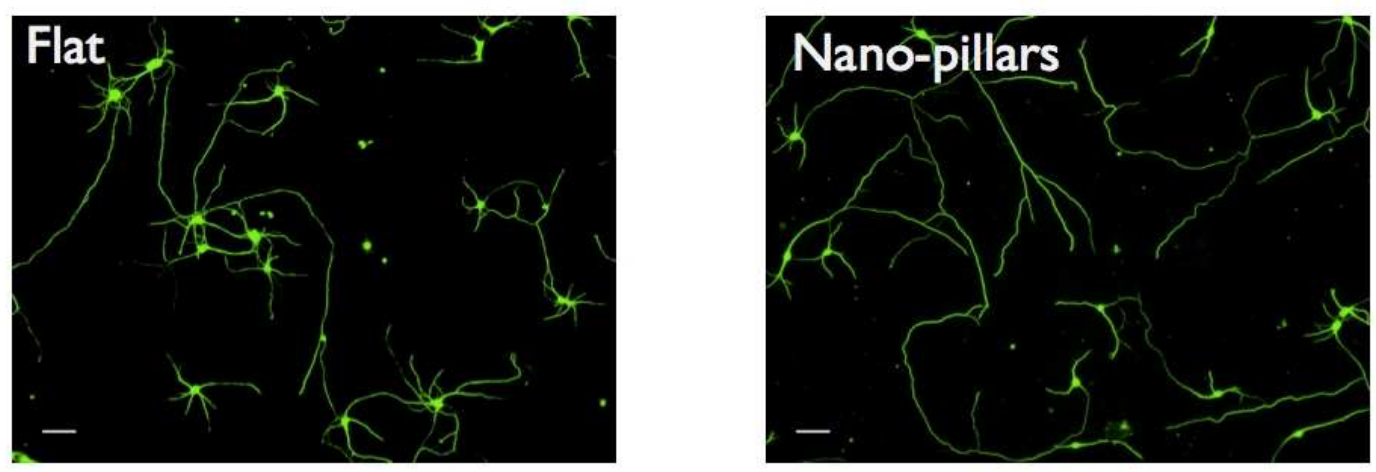

b
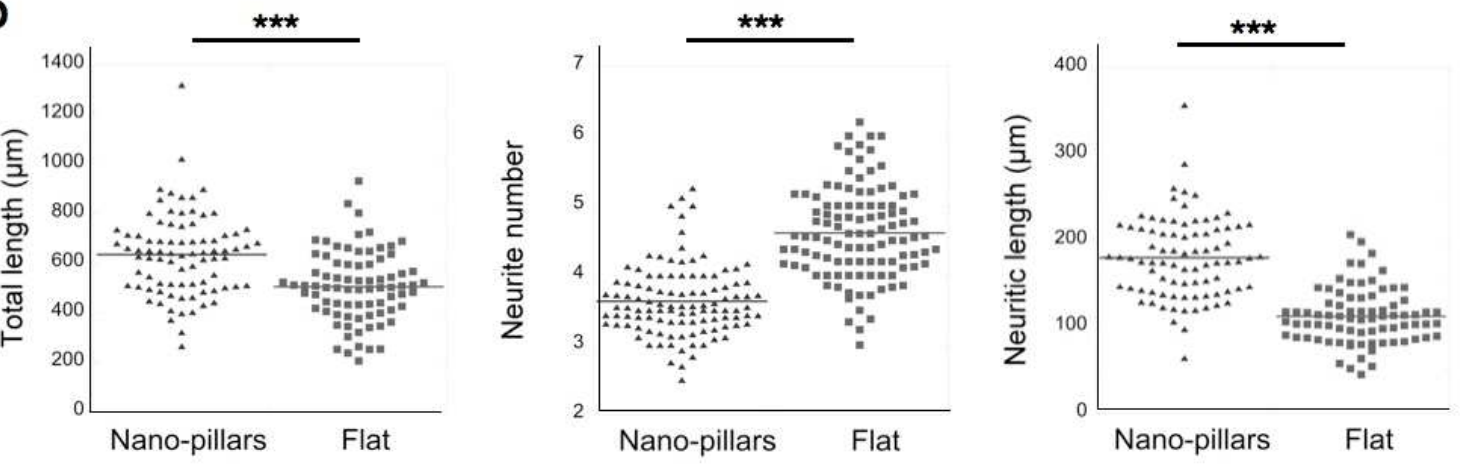

Figure 4 - Neuronal development at 3DIV on nano-pillared and flat silicon surfaces.

a) Example of neuronal development on each condition. Microtubules (YL1/2, green) immunolabellings. Scale bars: $50 \mu \mathrm{m}$.

b) Distribution of the total neurite length, the neurite number and the mean neurite length per 
cell ( $n=397$ for pillared surfaces and $n=353$ for flat surfaces, 3 cultures, 3 silicon substrates per culture) obtained through the analysis of about 80 optical micrographs per condition. The mean neurite length is calculated, for each micrograph, from the total length divided by the number of neurites $(* * *, p<0.001)$.

\section{2 - Neuronal development at early stages}

We found that, as for 3DIV, neurons developed longer neurites at 2DIV on nano-pillared as compared to flat surfaces. The total neurite length is now $438 \pm 76 \mu \mathrm{m}$ compared to $382 \pm 72 \mu \mathrm{m}$ $(\mathrm{p}<0.01, * *)$, the mean neurite length $125 \pm 22 \mu \mathrm{m}$ compared to $86 \pm 17 \mu \mathrm{m}(\mathrm{p}<0.001, * * *)$ (Figure 5a) for a neurite number of $3.5 \pm 1.4$ as compared to $4.6 \pm 1.4(\mathrm{p}<0.001, * * *)$. The number of neurites is, as expected, not significantly different from that measured in the 3DIV condition.

To figure out when the number of neurites diverges between the two types of substrates, we looked at the very first stage of development, i.e. 10 hours after plating (h10 condition). At this stage, newly formed neurites still cohabit with lamellipodia. In average, we counted 1.05 neurite and 1.2 lamellipodia on nano-pillars $(\mathrm{n}=86)$, and very similar values were obtained on flat silicon surface (1.09 neurite and 1.26 lamellipodia, n=99). We also measured the lamellipodia surface and obtained $71.6 \pm 39 \mu \mathrm{m}^{2}$ on nano-pillars compared to $81.2 \pm 50.6 \mu \mathrm{m}^{2}$ on flat surfaces. In addition, the length of the longest neurite in both conditions was not significantly different, with $25 \pm 11 \mu \mathrm{m}$ (nano-pillars, $\mathrm{n}=38$ ) and $24 \pm 14 \mu \mathrm{m}$ (flat surfaces, $\mathrm{n}=47$ ). No morphological differences were thus found between the two physical environments or, in other terms, nothing distinguishes neurites or lamellipodia that formed on nano-pillars or on flat surfaces at the very early stage of development. This may indicate that the differential development between nano-pillared and flat surfaces may occur only when all neurites are formed, presumably between h10 and 1DIV.

a

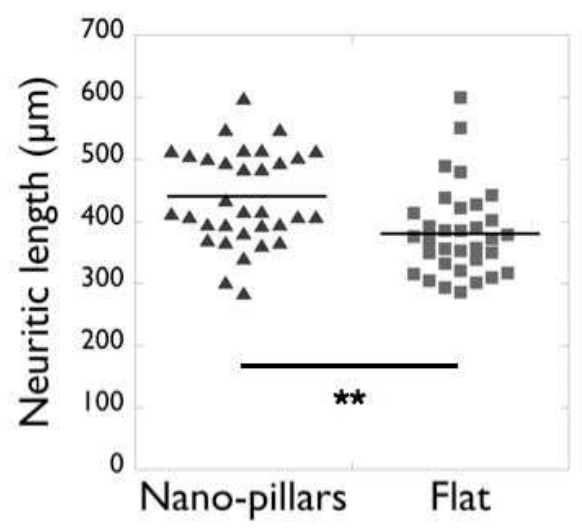

b

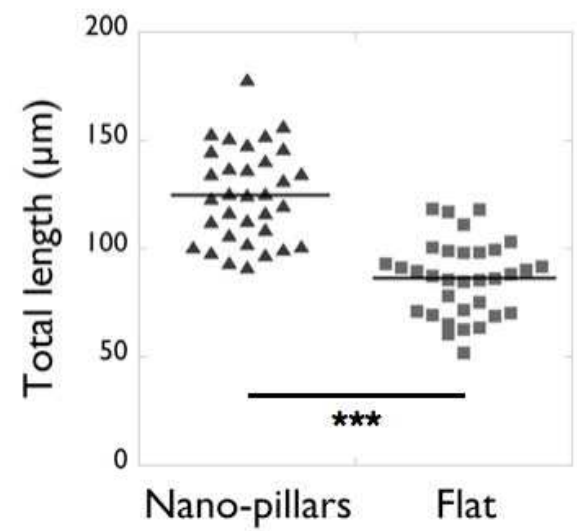


Figure 5 - Neuronal growth at 2DIV on nano-pillared and flat silicon surfaces

Distributions of the mean neurite length (a) and of the total length obtained at 2DIV; $n=397$ (nano-pillared surface) and $n=323$ (flat conditions). Data were obtained from the analysis of 33 optical micrographs per condition (1 culture, 2 silicon substrates) (**, p<0.01; ***, $\mathrm{p}<0.001)$.

\section{3 - Neuronal development on laminin}

The above results show that the surface topography can hasten the neurite elongation, even without any morphological guidance. Growth enhancement was previously described in the case of multiple adhesion proteins, including Laminin $(\mathrm{LN})^{21}$. These proteins generate specific signals, very different from the physical signal of nano-pillared surfaces, so it seemed interesting to coat our samples with LN on top of PLL to confront the effects of the two types of cell environment.

On both nano-pillared and flat topographies, neurons were more developed with a PLL/LN coating compared to PLL alone, confirming that LN is a strong growth enhancer. However, neurons on the pillars $+\mathrm{LN}$ surface were not more developed $(660 \pm 174 \mu \mathrm{m})$ than those on the flat $+\mathrm{LN}$ one $(773 \pm 138 \mu \mathrm{m})(\mathrm{n} \approx 200$ neurons per condition, 1 culture $)$. Interestingly, the number of neurites was not significantly different between the nano-pillared $(4.05 \pm 0.6$ neurites) and flat ( $3.85 \pm 0.5$ neurites) surfaces.

\section{4 - Influence of nano-pillars on axonal polarization}

\section{1 - Polarization rate}

Neuronal elongation on nano-pillars is accelerated. Knowing that the nascent axon is, as described in vitro, the longest process ${ }^{22}$, an accelerated elongation rate may affect the rate of polarization. The majority of neurons grown on poly-L-lysine (PLL) polarized within fortyeight hours after plating ${ }^{20}$. We thus counted the number of polarized neurons on nano-pillars and on flat surfaces coated with PLL at 2DIV ( 1 culture) and 3DIV ( 2 cultures). The difference between the two adhesive conditions is dramatic at $2 \mathrm{DIV}$ : a rate of $74.2 \%$ of polarization was obtained on nano-pillars ( $\mathrm{n}=260$ cells) while flat surfaces generated significantly less polarized neurons $(57.9 \%, \mathrm{n}=235, \mathrm{p}<0.01, * *)$. Yet, this disparity vanishes at 3 DIV where an asymptotic polarization is reached in both conditions, with $76.8 \%(\mathrm{n}=164$ cells $)$ and $74.7 \%(n=95)$ for the polarization rate on nano-pillared and flat silicon surfaces, respectively.

Nano-pillars thus increase the rate of neurite elongation and polarization. In this context, we looked at the polarization when the two physical environments were simultaneously available 
for a given cell. 


\section{2 - Polarization at frontiers}

As the elongation rate is enhanced on nano-pillars, the question of a selectivity of this weakly adhesive environment toward axonal differentiation is an open and sound question. To answer it, the notion of neighborhood regarding the boundary between nano-pillars and flat surfaces must be specified to select the pertinent population of « boundary neurons ».

The mean neurite length before polarization has been estimated to $35 \mu \mathrm{m}^{21}$. In this context, a simple selection rule for a «boundary neuron » is that at least one of its neurites must have crossed that boundary before reaching a length of $35 \mu \mathrm{m}$ (see the sketch of Figure 6b). In other terms, neurons are selected such as the distance between their soma and the border along the path followed by at least one neurite does not exceed $35 \mu \mathrm{m}$. Neurons are then classified according to the side of the border where are the somas, and the side where the axons are found (one example of such neurons is given in Figure 6a), and results are reported in Table 1 (see also Figure 6c).

\section{Table 1}

\begin{tabular}{|l|l|l|l||l|}
\cline { 3 - 4 } \multicolumn{2}{c|}{} & $\begin{array}{l}\text { Soma on nano- } \\
\text { pillars }\end{array}$ & $\begin{array}{l}\text { Soma on flat } \\
\text { surfaces }\end{array}$ & $\begin{array}{l}\text { Total number of } \\
\text { cells }\end{array}$ \\
\hline 2DIV & $\begin{array}{l}\text { Number of boundary } \\
\text { neurons }\end{array}$ & 33 & 31 & 64 \\
\cline { 2 - 4 } & $\begin{array}{l}\text { Percentage (number) of } \\
\text { axons on nano-pillars }\end{array}$ & $67 \%(22)$ & $45 \%(14)$ & $56 \%(36)$ \\
\hline 3DIV & $\begin{array}{l}\text { Number of boundary } \\
\text { neurons }\end{array}$ & 46 & 38 & 84 \\
\cline { 2 - 4 } & Axons on nano-pillars & $85 \%(39)$ & $66 \%(25)$ & $76 \%(64)$ \\
\hline $\begin{array}{l}\text { Theoretical percentages of axons on } \\
\text { nano-pillars }\end{array}$ & $60 \%$ & $40 \%$ & \\
\hline
\end{tabular}

Table - Axonal localization of boundary neurons for soma located on each side of the frontier separating nano-pillared and flat surfaces ( 1 culture). 

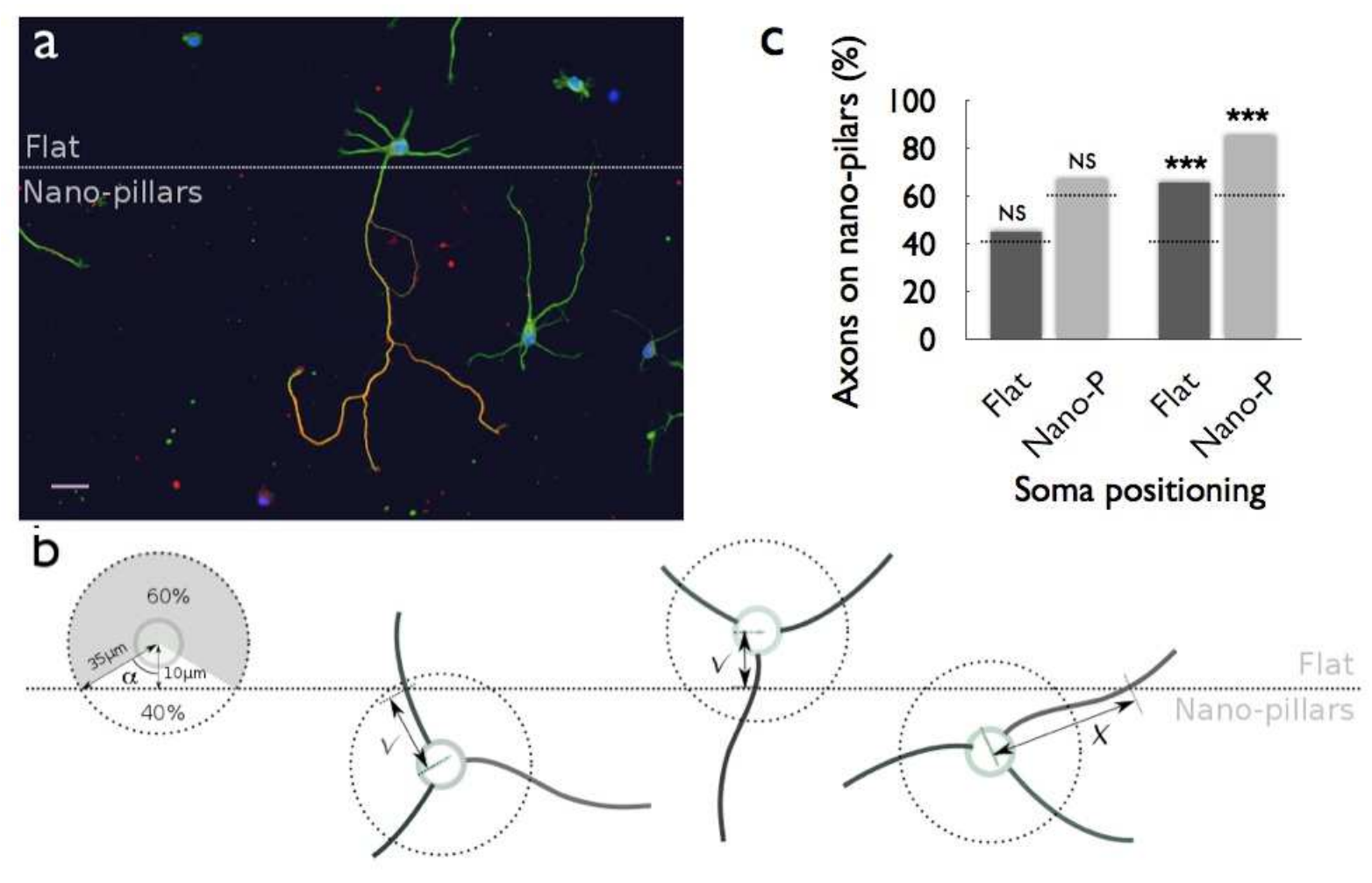

Figure 6 - Axonal polarization at frontiers.

a) Optical micrograph of a neuron crossing the border between the flat and nano-pillared surfaces. Microtubules (YL1/2, green), axon (Tau, red) and nucleus (Hoechst, blue) immunolabellings. Scale bar : $25 \mu \mathrm{m}$.

b) Sketch representing the rule of selection of "boundary neurons" from the use of a threshold in neurite length of $35 \mu \mathrm{m}$ between the soma center (soma are represented by green circles) and the frontier between the nano-pillared and flat surfaces (see text). The abbreviations and $\mathrm{X}$ identify selected or rejected neurons, respectively.

c) Percentage of axons at 2 and 3 DIV found exclusively on nano-pillared surfaces for soma localization on either side of the frontier, i.e. on either flat and nano-pillared (nano-P) surfaces. Dashed lines denote the theoretical percentage expected without any influence of the frontier $(\mathrm{p}<0.001, * * *, \mathrm{NS}$ : non significant). See table 1 for the numerical values attached to each condition.

Then, to estimate the theoretical percentage of axons on both sides in the case this composite adhesive environment would not affect the localization of axonal specification, we first measured the average distance between the soma and the frontier in the population of selected neurons. This experimental average distance was $10 \mu \mathrm{m}$. We then draw a $35 \mu \mathrm{m}$ radius circle whose center, modeling the position of a soma, is localized at 10 microns from the boundary (see the sketch in Figure 6b for a visual description of our procedure) while the circle represents the border a neurite should be the first to cross to become the axon. In the absence of any bias provided by the environment, the axon should go beyond that circle in a random 
orientation. Thus, for a soma located on the flat surface, the ratio of the perimeter included in the nano-pillar $\left(p_{N P}\right)$ area on the total perimeter $\left(p_{T}\right)$ gives the theoretical percentage of axons expected in the weakly adhesive area in the absence of any bias. Numerically, this percentage $p$ can be written as (see Figure 6 for the signification of $\alpha$ ):

$p=\frac{p_{N P}}{p_{T}}=\frac{2 \alpha \times R}{2 \pi R}=\frac{\operatorname{arcos}\left(\frac{10}{35}\right)}{\pi} \approx 0.40$, or $40 \%$

with $R=35 \mu \mathrm{m}$. Conversely, the percentage of axons expected in the flat area in the absence of any bias is $60 \%$. The same reasoning applies for a soma located on the nano-pillared area.

The comparison of experimental results to the theoretical percentage (Table 1) demonstrates that there is a significant axonal preference at 3 DIV for nano-pillared areas for soma being on either side of the boundary $(\mathrm{p}<0.001, * * *$, Figure $6 \mathrm{c})$.

\section{3 - Remark: axonal versus dendritic length}

Nano-pillars increase the rate of neurite elongation and polarization. A similar phenomenon was observed on flat coverslips using laminin instead of poly-L-lysine. However, laminin is known to selectively enhance the axonal elongation, and both the number of neurites and the length of the other processes are unchanged ${ }^{20}$. We therefore checked the relative length of the axons that grow on nano-pillared and on flat silicon surfaces and compared these values to the total length of the neurites per cell. On average, an axon on top of nano-pillars displays the same increase of length (around 22\%) as the whole neurite network, indicating a non-specific effect of these nanostructures regarding axonal elongation. In conclusion, the dendritic length seems to compensate the reduction in the number of neurites observed on nano-pillars, so that the total length of the two poles of the cell (axon / dendrites) retain the same proportion of lengths.

\section{5 - Discussion}

\section{1 - About the development of neurites on nano-pillars}

Neurite elongation on nano-pillars is accelerated. Despite the high rigidity of bulk silicon (in the range of hundreds of GPa), strongly bended nano-pillars are sometimes observed (see for example the pillars located in the boxed area of Fig. S3). Therefore we might consider that nano-pillars would display lower effective rigidities than bulk silicon due to their high aspect ratio. Cells in general are very sensitive to their mechanical environment, and it has been reported a dramatic change in neurite number and length below a rigidity of $100 \mathrm{~Pa}$ in the 
PC12 neuronal cell line ${ }^{23}$ as well as an increase of branching on very soft gels ${ }^{24}$. The relationship between the Young modulus of the bulk material that composes a rod $\left(E_{b u l k}\right)$ and the apparent Young modulus of the rod itself $\left(E_{\text {eff }}\right)$ is a function of its radius and length and takes the form:

$$
E_{\text {eff }}=\frac{27}{16} E_{b u l k}\left(\frac{R}{H}\right)^{3},
$$

with $\mathrm{H}$ the height and $\mathrm{R}$ the radius of the rod (see Supporting Material S4 for details).

Nano-pillars are composite structures made of silicon $\left(E_{S i}=170 \mathrm{GPa}\right)$ and silicon dioxyde ( $E_{\mathrm{SiO}_{\mathbf{2}}}=50 \mathrm{GPa}$ ) produced by the etching process. From the geometrical parameters of nanopillars, i.e. $H=700 \mathrm{~nm}$ and $R=35 \mathrm{~nm}$ (taking the average between the mean bottom and top radius of the nano-pillars), we obtain $E_{\text {eff }}=10.5 \mathrm{MPa}$ using the lowest Young modulus, i.e. $E_{\mathrm{SiO}_{\mathbf{2}}}$. Although this value is much lower than any bulk values, this rigidity remains in a range known to have no effect on cortical neuron development ${ }^{25}$. Therefore, even by considering the extreme situation where the nano-pillars might display a linear elastic behavior in a large range of deformations, the changes we observed in neuronal elongation do probably not result from the flexibility of nano-pillars.

An accelerated directional neurite elongation was reported on sub-micrometric grooves formed on polyurethane acrylate substrates, providing a one-dimensional anisotropic adhesive environment to cells ${ }^{26}$. This phenomenon was associated to the presence of a stable, fully adherent filopodia population aligned with the grooves and a relative destabilization of the perpendicular filopodia that experience the substrate striations transversally. On micropillared surfaces, the faster axonal elongation was correlated to a decrease of the overall growth cone size, probably due to a narrowing of this structure that occurs between pillars. This result was explained in terms of possible boosts of growth at pillar contacts ${ }^{6}$. In our study, it is interesting to note that, although directional choices are made by neurites in their positioning on the top of nano-pillars, the accelerated elongation that occurs in this somehow point-like, zero-dimensional adhesive configuration is observed in the absence any channeling effect such as the one provided by the one-dimensional topography of grooves or the presence of micro-pillars. Our results suggest that the primary signal of accelerated elongation might be given by the population of transverse, weakly adherent filopodia that sense the presence of lateral topographies provided either by micro- or nano-pillars and grooves, and that this signal may have a significant effect without even altering the overall shape of growth cones. 
Chemical and topographic environments are probably not sensed by neurons the same way. Pillars produce a physical signal, intrinsically linked with their spatial organization and therefore sensed "as a whole". On the contrary, chemical cues like laminin produces a specific signal at the molecular level, which allows it to induce a modulated response depending on its concentration $^{20}$ or its surface density (in the present work, the effective coating density is reduced by an order of magnitude by nano-pillared compared to flat surfaces). Considering furthermore that the two different signals may also activate competing signaling pathways, we face a rather complex situation ruling out the possibility of a simple additive effect between chemical and topographic cues. Interestingly, nano-pillars combined with a laminin coating do not induce a reduction in the number of neurites, in contrast to what is observed in nonspecific adhesion condition (i.e. with PLL coating). This result is surprising knowing that laminin increases only the length of the axon, and therefore should not affect the growth dynamics of undifferentiated neurites in the first stage of development. Although puzzling, neuronal growth on nano-pillars reveals that the mode of action of laminin is more complex than expected, since it may regulate the number of neurites too.

Last, the clear reduction of the neurite number on PLL-coated nano-pillars combined with the absence of morphological differences after 10 hours in vitro suggests that only the last stages of neurite formation following the partial collapse of the primary lamellipodium around the cell body ${ }^{27}$ are affected by topographic cues.

The mechanisms at the origin of the accelerated neurite elongation provided by the fragmentation of adhesive surfaces are still unknown, and only speculative hypothesis could be formulated at this stage. Among them, an increase of neurite tension, resulting from the spacing between adhesion points could be pertinent for two reasons: stretching neurites results in an accelerated elongation that eventually lead to their differentiation into axons ${ }^{28}$, and axons displayed periodic actin rings wrapped around their circumference spaced by a submicrometric distances ${ }^{29}$. These actin rings may have a role in sustaining the mechanical strains to which axons are subjected. In our work, we have identified discrete actin/vinculin structures whose spacing might be imposed by the distance between adhesive structures provided by the contact of neurites with the top of nano-pillars. An increase of neurite tension mediated by these discrete actin structures is a hypothesis that should deserve specific investigations in a future work. 



\section{2 - Consequence of nano-pillars on axonal polarization}

The accelerated elongation rate on nano-pillars is accompanied by a preferential axonal localization for cells located close to the flat/nano-pillar frontiers. Note that this effect was not yet significant after 2DIV, which might indicate that neurons that polarize later show an higher sensitivity to their environment, presumably because they already spent more time to explore it.

But neurites crossing this frontier are also confronted to a step of $2 \mu \mathrm{m}$ height, as shown in Figure 1a. The work of Li and Folch ${ }^{30}$ has shown that a step of 2.5 microns separating two flat areas is not sufficient to localize the axons. The step height has to reach the value of $11 \mu \mathrm{m}$ to prevent the passage of $50 \%$ of the axons, regardless of the crossing direction. These results were interpreted as a resistance to curvature, leading to the choice of a path that minimizes axonal bending: according to the step height, an axon may prefer to avoid crossing the step and bend along the frontier. From these results, the $2 \mu \mathrm{m}$ steps that separates the nano-pillars from the flat areas should not have significant influence, especially as these steps are not purely vertical, which reduces the curvature necessary for their crossing. In addition, a possible influence of the $2 \mu \mathrm{m}$ step height difference would be symmetrical: neurons on flat areas should have more axons on this side of the frontier and inversely. We observe on the contrary more axons on nano-pillars compared to the theoretical estimations, regardless of the soma location. The effect of the steps themselves, if any, is therefore fully dominated by the nano-pillars selective environment itself.

Let us note that finding more axons on a surface that increases the elongation rate is consistent with the notion proposed by Craig et al. ${ }^{31}$, and further explored with the use of micropatterns ${ }^{32}$, of a process of axonal specification triggered when the first undifferentiated neurite exceeds a threshold of length.

\section{6- Conclusion}

We have shown in this work that a change from uniform to discontinuous adhesive conditions on the nanometric scale influences many aspects of neuronal growth. Our results show that neurite elongation is somehow guided but most of all accelerated under the weak adhesion conditions provided by silicon nano-pillared surfaces, leading to a preferential localization of axonal specification.

This major influence of the physical environment raises important questions relative to the mechanism of neuritogenesis and neurite elongation that should motivate further studies. So far, the nano-pillared surfaces used in this study were obtained using a simple plasma etching process. However, the recent work of $\mathrm{Xu}$ et al. ${ }^{29}$, by revealing the existence of periodic actin 
rings in the axon, should motivate the search of periodic cellular adhesive complexes associated to these actin/vinculin structures. Thus, surfaces characterized by a precise control of the inter-nano-pillars distance would be very useful, and their implementation on either silicon or PDMS (to explore a wider range of effective rigidities) substrates is a natural perspective, although technologically challenging, of the present work.

\section{Acknowledgements}

The authors thank A. Schweitzer and N. Collomb for excellent technical support with neuronal cultures, the NanoFab team for their support and advices concerning silicon etching, and C. Tomba for vinculin stainings. This work has been funded by the Fondation Nanoscience, Grenoble (France).

\section{Supporting information available}

This information is available free of charge via the Internet at http://pubs.acs.org/. 


\section{References}

(1) Ghashghaei HT, Lai C, and Anton ES. Neuronal migration in the adult brain: are we there yet? Nat Rev Neurosci. 2007, 8, 141-151.

(2) Robles E, and Gomez TM 2006. Focal adhesion kinase signaling at sites of integrinmediated adhesion controls axon pathfinding, Nature Neuroscience 9(10) 1274-83. doi:10.1038/nn176

(3) Geiger B, Spatz JP, Bershadsky AD 2009. Environmental sensing through focal adhesions, Nature Reviews Molecular Cell Biology 10(1):21-33.

(4) Dowell-Mesfin NM et al. 2004. Topographically modified surfaces affect orientation and growth of hippocampal neurons, J. Neural Eng. 1, 78-90

(5) Kundu A et al. 2013. Superimposed topographic and chemical cues synergistically guide neurite outgrowth, Lab Chip 13, 3070-3081.

(6) Micholt L, Gärtner A, Prodanov D, Braeken D, Dotti CG, et al. (2013) Substrate Topography Determines Neuronal Polarization and Growth In Vitro. PLoS ONE 8(6): e66170. doi:10.1371/journal.pone.0066170.

(7) Fozdar DY, Lee JY, Schmidt CE and Chen S 2010. Selective axonal growth of embryonic hippocampal neurons according to topographic features of various sizes and shapes, International Journal of Nanomedicine 2011:6 45-57.

(8) Spatz JP and Geiger B 2007 Molecular Engineering of Cellular Environments: Cell Adhesion to Nano-Digital Surfaces, Methods Cell Biol. 83, 89-111.

(9) Hällström W, Prinz CN, Suyatin D, Samuelson L, Montelius L and Kanje M 2009. Rectifying and Sorting of Regenerating Axons by Free-Standing Nanowire Patterns: A Highway for Nerve Fibers, Langmuir 25(8), 4343-4346.

(10) Piret G, Perez MT, Prinz CN 2012. Neurite outgrowth and synaptophysin expression of postnatal CNS neurons on $\mathrm{GaP}$ nanowire arrays in long-term retinal cell culture, Biomaterials 34(4):875-87.

(11) Prinz C, Hällström W, Martensson T, Samuelson L, Montelius L and Kanje M 2008. Axonal guidance on patterned free-standing nanowire surfaces, Nanotechnology 19 (2008) 345101 .

(12) Robinson JT, Jorgolli M, Shalek AK, Yoon MH, Gertner RS, and Park H. 2012. Vertical nanowire electrode arrays as a scalable platform for intracellular interfacing to neuronal circuits', Nature Nanotech. 7 (3) 180-184.

(13) Xie C, Lin Z, Hanson L, Cui Y, and Cui B 2012. Intracellular Recording of Action Potentials by Nanopillar Electroporation, Nat Nanotechnol 7, 185-190. doi:10.1038/nnano.2012.8.

(13) Roth S, Bugnicourt G, Bisbal M, Gory-Fauré S, Brocard J, Villard C 2012, Neuronal 
Architectures with Axo-dendritic Polarity above Silicon Nanowires, Small 8(5), 671-675.

(14) Bray DF, Bagu J, Koefler P 1993, Comparison of Hexamethyldisilazane (HMDS), Peldri II, and critical-point drying methods for scanning electron microscopy of biological specimens, Microscopy research and technique 26, 489-495.

(15) Abràmoff MD, Magalhães PJ, and Ram SJ 2004. Image processing with imageJ. Biophotonics international, 11(7) :36-42.

(16) Nečas A, Klapetek P, Gwyddion: an open-source software for SPM data analysis, Cent. Eur. J. Phys. 10(1) (2012) 181-188

(17) Zhang TY and Suen CY 1984. A fast parallel algorithm for thinning digital patterns. Communications of the ACM, 27(3) 236-239.

(18) Katz MJ 1985, How Straight Do Axons Grow?, J. Neurosci. 5 (3) 589-595.

(19) Carlstrom L P, Hines J H, Henle S J and Henley J R 2011, Bidirectional remodeling of b1-integrin adhesions during chemotropic regulation of nerve growth BMC Biology, 9 (82).

(20) Lein PJ, Banker GA, Higgins D 1992. Laminin selectively enhances axonal growth and accelerates the development of polarity by hippocampal neurons in culture, Brain Res Dev 69(2) 191-197.

(21) Esch T, Lemmon V, and Banker G 1999. Local presentation of substrate molecules directs axon specification by cultured hippocampal neurons. J Neurosci 19(15) 6417-26.

(22) Dotti CG, Sullivan CA, Banker GA 1988. The establishment of polarity by hippocampal neurons in culture, J. Neuroci. 8, 1454.

(23) Leach JB, Brown XQ, Jacot JG, DiMilla PA and Wong JY 2007. Neurite outgrowth and branching of PC12 cells on very soft substrates sharply decreases below a threshold of substrate rigidity, J. Neural Eng. 4, 26-34

(24) Flanagan LA, Ju YE, MargB, Osterfield M, Janmey PA 2002. Neurite branching on deformable substrates. Neuroreport. 13 (18):2411-5.

(25) PC Georges, Miller WJ, Meaney DF, Sawyer ES and Janmey PA 2006. Matrices with Compliance Comparable to that of Brain Tissue Select Neuronal over Glial Growth in Mixed Cortical Cultures, Biophys J. 90(8) 3012-3018, doi: 10.1529/biophysj.105.073114

(26) Jang KJ, Kim MS, Feltrin D, Jeon NL, Suh KY, and Pertz O 2010. Two distinct filopodia populations at the growth cone allow to sense nanotopographical extracellular matrix cues to guide neurite outgrowth. PLoS One 5(12):15966.

(27) Dehmelt L and Halpain S 2004. Actin and Microtubules in Neurite Initiation: Are MAPs the Missing Link? J Neurobiol. 58(1) 18-33.

(28) Lamoureux P, Ruthel G, Buxbaum RE and Heidemann SR 2002. Mechanical tension can specify axonal fate in hippocampal neurons $J$ Cell Biol 159(3) 499-508 doi/10.1083/jcb.200207174 
(29) Xu K, Zhong G, Zhuang X 2013. Actin, Spectrin, and Associated Proteins Form a Periodic Cytoskeletal Structure in Axons, Science 25, 452-456.

(30) Li N and Folch A 2005. Integration of topographical and biochemical cues by axons during growth on microfabricated 3-d substrates. Exp Cell Res 311(2) 307-316.

(31) Craig AM, Jareb M, and Banker G 1992. Neuronal polarity. Curr Opin Neurobiol 2(5) :602-606.

(32) Yamamoto H et al., 2013. Differential neurite outgrowth is required for axon specification by cultured hippocampal neurons, J Neurochem. 123(6): 904-910. 